\title{
Why optics students should take digital signal processing courses and why digital signal processing students should take optics courses
}

\section{W. Thomas Cathey}

W. Thomas Cathey Jr., "Why optics students should take digital signal processing courses and why digital signal processing students should take optics courses," Proc. SPIE 3831, Sixth International Conference on Education and Training in Optics and Photonics, (16 June 2000); doi: 10.1117/12.388706

Event: Education and Training in Optics and Photonics (ETOP'99), 1999, Cancun, Mexico 


\title{
Why optics students should take digital signal processing courses and why digital signal processing students should take optics courses
}

\author{
W. Thomas Cathey * \\ Imaging Systems Laboratory, Dept. of Electrical and Computer Engineering \\ University of Colorado, Boulder, Colorado 80309-0425
}

\begin{abstract}
This paper is based on the claim that future major contributions in the field of imaging systems will be made by those who have a background in both optics and digital signal processing. As the introduction of Fourier transforms and linear systems theory to optics had a major impact on the design of hybrid optical/digital imaging systems, the introduction of digital signal processing into optics programs will have a major impact. Examples are given of new hybrid imaging systems that have unique performance. By jointly designing the optics and the signal processing in a digital camera, a new paradigm arises where aberration balancing takes into consideration not only the number of surfaces and indices of refraction, but also the processing capability.
\end{abstract}

Keywords: Hybrid digital/optical imaging systems, aberration balancing, education, digital signal processing

\section{INTRODUCTION}

The introduction of linear systems theory and Fourier transforms into optics changed the way that many imaging systems were designed and evaluated. One example is the use of modulation transfer functions based on the concept of spatial frequencies to assess the quality of an imaging system. Digital signal processing has been used for such things as edge enhancement, and other operations that are now commonly done with software such as Adobe Photoshop. This paper examines what changes should be made in the design of the optics portion of imaging systems if one knows that signal processing will be done on the intermediate image that is acquired by the optics. The final image is obtained only after signal processing. So, the question should be asked "How should the optics of the hybrid optical image acquisition and signal processing system be modified so that the desired signal processing is easier or, indeed, even possible?" Examples are given of two hybrid imaging systems that have capabilities that would not be possible if the hybrid imaging system were not designed as a whole. That is, the performance of those systems could not be obtained with a conventional imaging system followed by any form of signal processing. Then, an example is given of how aberration balancing in a hybrid optical/digital system leads to dramatically different imaging systems. Finally, the prediction is made that many new, different imaging systems will be created by students who are educated in both optics and digital signal processing.

\section{BACKGROUND}

In the 1960's the ideas that are common to linear systems theory were becoming commonly used in the design and analysis of optical systems. ${ }^{1}$ Even though imaging systems have identical outputs over only limited regions of the image, the use of spatial frequencies and optical transfer functions became common in evaluating imaging systems. The mathematical tools that were developed for communications theory were directly applicable to optical systems by replacing temporal frequencies in cycles per second by spatial frequencies in cycles per millimeter. An important difference between temporal systems and spatial systems is that the spatial systems are not causal. That is, the result can be at a negative value of $x$, even if the cause is at a positive value of $x$. Impulse responses can be symmetric in spatial systems. This can not happen if the variable is time. The fact that imaging systems are two dimensional requires that spatial frequencies be used in two dimensions. This has the effect that some limitations that are encountered with one-dimensional temporal frequencies are not present when the system has two-dimensional spatial frequencies. One example is phase retrieval from amplitude information; the extra dimension lends "support" that makes the problem more tractable.

* Correspondence: e-mail: cathey@colorado.edu; Web: http://www.colorado.edu/isl; Telephone: (303) 492-1888; Fax: (303) $492-5810$ 
The emphasis in signal processing research for the last few decades has concentrated on digital signal processing (DSP). However, the impact of DSP on imaging systems has been primarily in the area of processing images after they have been acquired by conventional imaging systems. A conventional imaging system is here defined as one that, in its design, no account is taken of what, if any, signal processing will be done on the recorded image. Examples of uses of DSP are increasing contrast, removing certain types of image noise, changing the effective gamma of the recording, etc. However, after the image is recorded, some information is irretrievably lost. No amount of processing will reclaim it. The real impact of DSP on imaging systems, I believe, is just beginning. The major benefits of DSP in imaging systems will occur when the complete imaging system (optics and DSP) is designed as an integrated, hybrid optical/digital system to achieve specified design goals. The need for this was pointed out several years ago ${ }^{2}$, but little progress was made.

Recently, some of the concepts of radar theory have been introduced into imaging theory. 3.4 The paper by Brenner, Lohmann, and Ojeda-Castaneda has been particularly useful in pointing out that the ambiguity function, which in radar is used to show signal strength as a function of Doppler and range, can be used to show the modulation transfer function of an imaging system as a function of misfocus. The ambiguity function has been useful in designing some hybrid optical/digital imaging systems. ${ }^{5}$ There are other ways of visualizing MTF as a function of misfocus, but the advantage of the ambiguity function is that optical systems designers can use the results of decades of work on the ambiguity function by researchers in radar. ${ }^{6}$ The following sections show two hybrid systems that were designed to perform specific functions. Then, an example is given to demonstrate the capabilities of hybrid systems to perform general imaging with many fewer optical elements, some of the work load being carried by DSP.

\section{HYBRID OPTICAL/DIGITAL IMAGING SYSTEM WITH VERY LARGE DEPTH OF FIELD}

Figure 1 shows a schematic diagram of one form of a hybrid imaging system. A special "wavefront mask" is placed in a principal plane of the lens system, an intermediate image is detected electronically, and then digital signal processing is done to obtain the final image 5. 7.8. In the example of extending the depth of field or focus that is discussed below, the wavefront mask is a phase plate that has a thickness, $t$, that varies as defined by $t=\alpha\left(x^{3}+y^{3}\right)$ where $\alpha$ is a constant. The

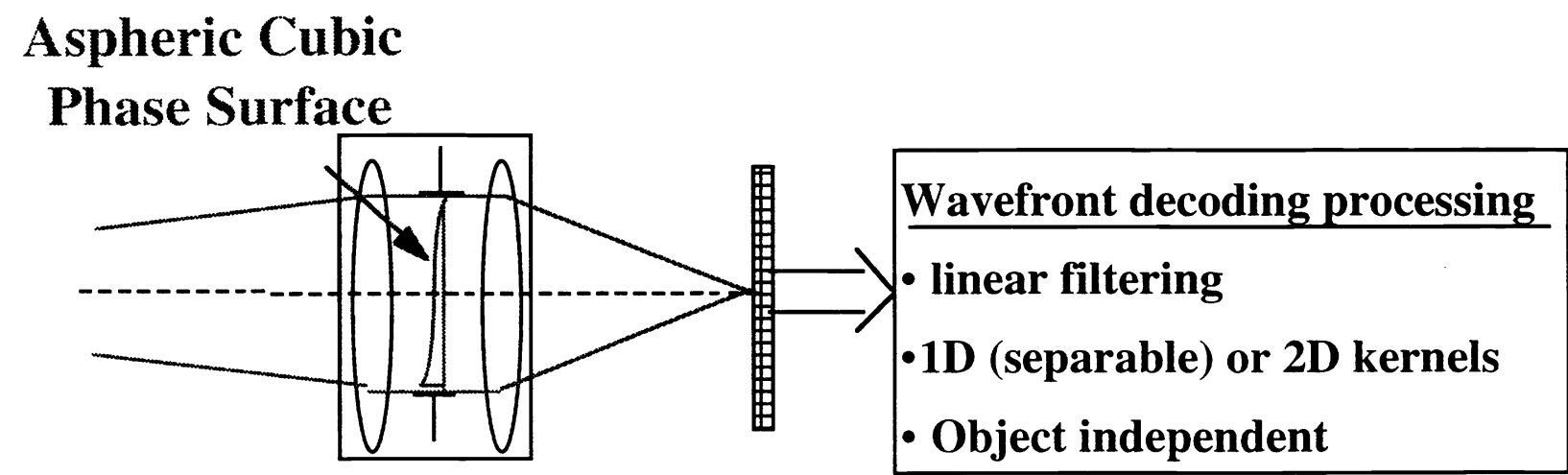

Fig. 1. Schematic of hybrid optical/digital imaging system. The intermediate image is acquired by the detector array, and the final image is produced by signal processing.

effect of the phase plate can be seen in Fig. 2 to cause the rays not to come to a focus as in a conventional system, but to provide a uniform density of waves in the vicinity of the focus region. Note the differences in scale between Fig. 2(a) and Fig. 2(b). The effect of this change in rays is to modify the point spread function (PSF), or impulse response, and the modulation transfer function of the optical portion of the imaging system as shown in Fig. 3 . This process has been referred to as "wavefront coding," meaning that the wavefront coming from the optics has been coded so that it is invariant to misfocus. An analogy is when an electrical signal such as a pulse is coded to be invariant to dispersion, for example, in the transmission path. After the electrical signal is transmitted and detected, it is then decoded to restore the pulse shape. In the case of an imaging system with wavefront coding, the detected signal is processed to decode the wavefront so that the MTF 
that is shown in Fig. 3 is converted to the desired MTF, such as that of a perfect imaging system. This signal processing introduces noise, and has been discussed elsewhere. ${ }^{9}$

Figure 4 shows the images of a bar target, inclined at $60^{\circ}$, as imaged by a conventional imaging system and by the hybrid system. The image formed by the conventional system is shown in Fig. 4(a), and the final image of the hybrid imaging system is shown in Fig. 4(c). Figure 4(b) shows a trace through the two images. Note that the misfocus of the conventional image is so severe that there is a contrast reversal in the image. The depth of field of the hybrid system is about eight times that of the conventional system. No signal processing can make the image in Fig. 4(a) look like the one in Fig. $4(\mathrm{c})$.

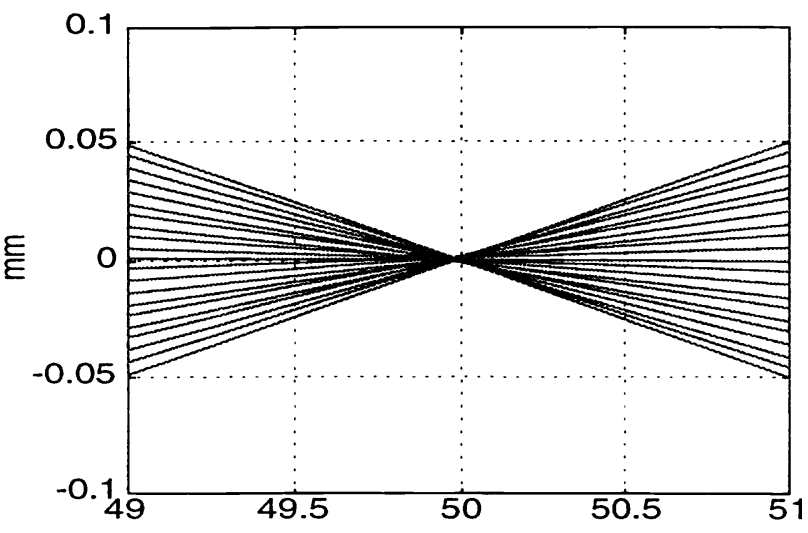

(a)

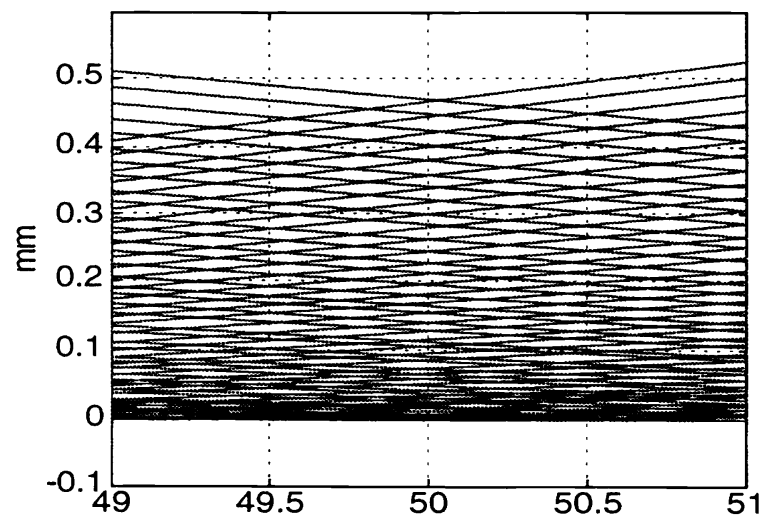

(b)

Fig. 2. Ray traces for (a) conventional lens, and (b) effect of the phase plate on the ray trace. Note the change in the vertical scale.
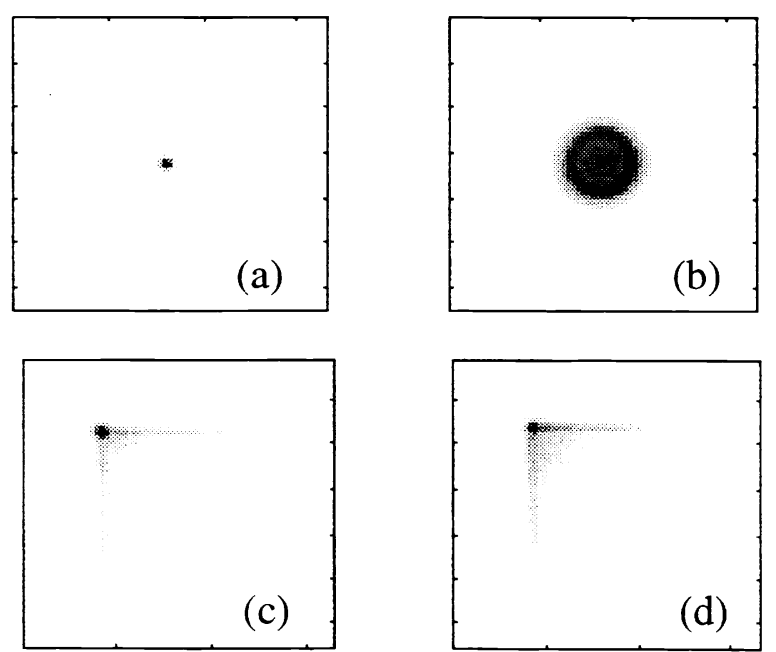

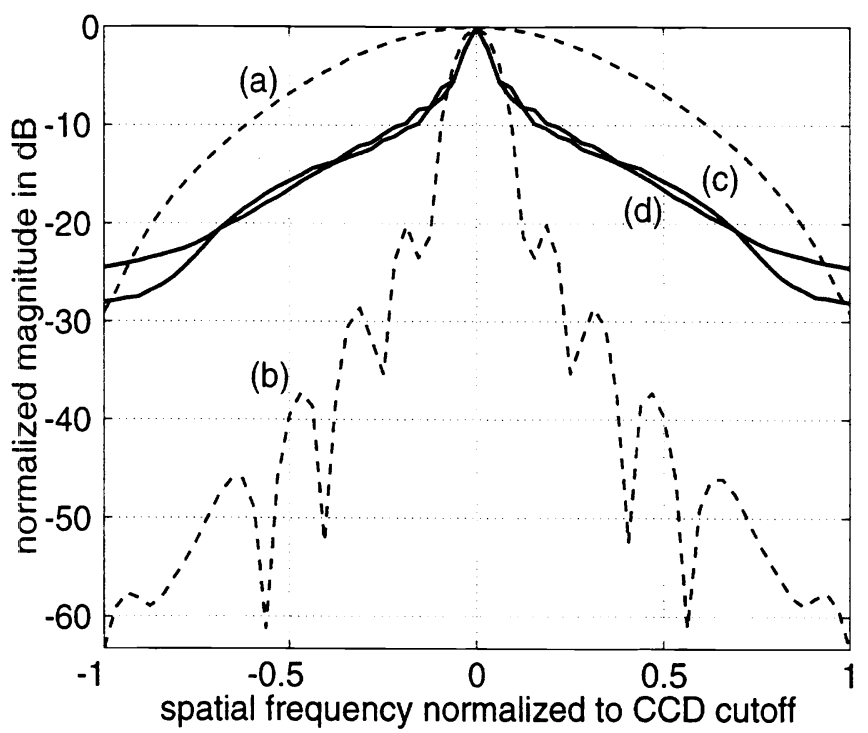

Fig. 3. Measured values of the PSFs and the MTFs for a conventional imaging system (a and $b$ ) and for the optical portion of the hybrid system ((c and d) of Fig. 1. The corresponding MTFs are shown to the right. 


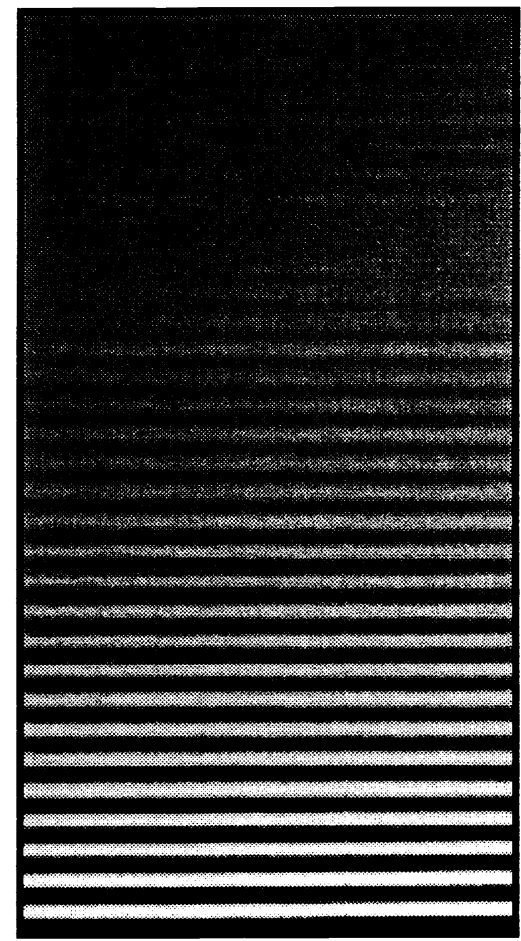

(a)

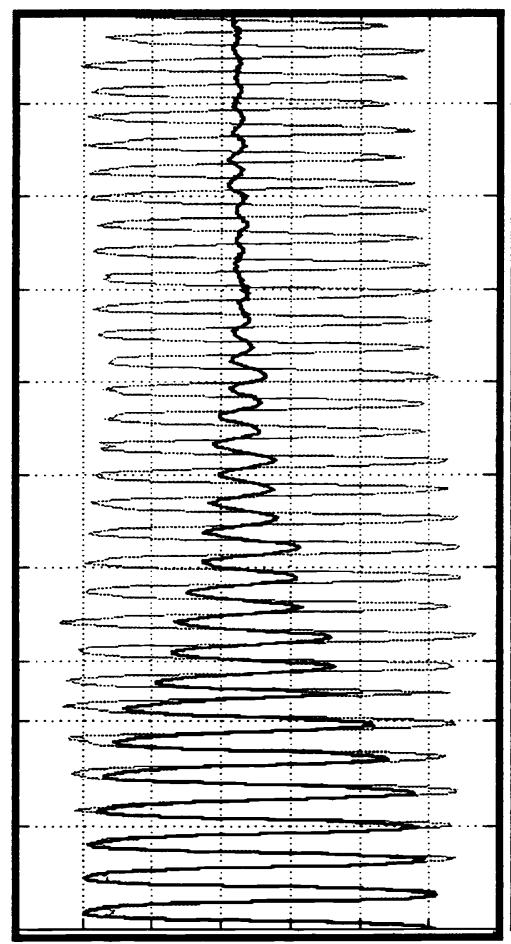

(c)

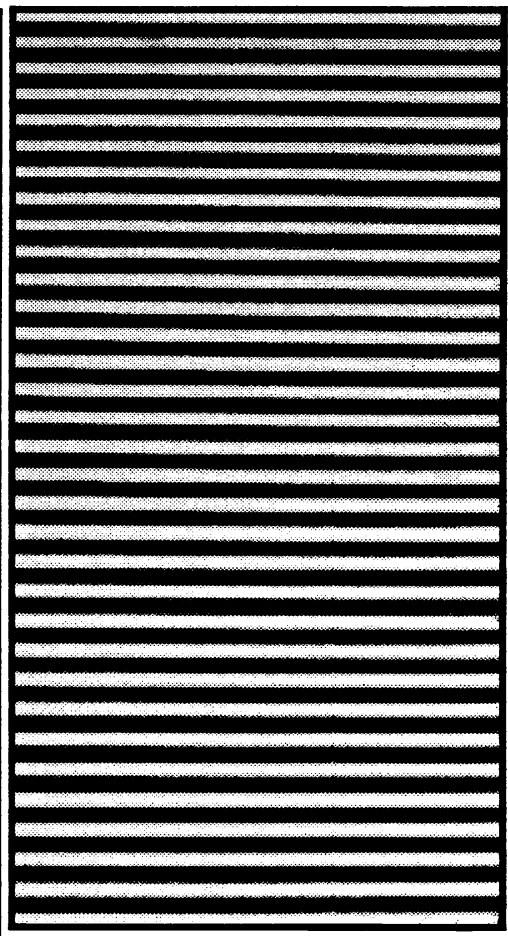

(c)

Fig. 4. Images of a bar pattern that has been inclined by $60^{\circ}$. The camera was focused on the bottom of the bar pattern. The left image was formed by a conventional imaging system. The right image was formed by a hybrid imaging system to extend the depth of field. The traces in (b) are through the center of the two images of a bar. Note the contrast reversal of the image formed with a conventional system.

\section{HYBRID OPTICAL/DIGITAL IMAGING SYSTEM FOR PASSIVE DETERMINATION OF DISTANCE}

Different wavefront coding masks can be used to make an imaging system that can be used to preserve and enhance information concerning the distance to objects in the field. ${ }^{10 .} 11$ The optical transfer function (OTF) is modified so that it changes with misfocus in specified ways. Then, if the object being imaged has an adequately wide spatial frequency spectrum, the important characteristics of the OTF also show up in the spatial frequency spectrum of the image. DSP can be used to extract the change in the image spectrum to determine the range or distance to the object in the scene. The transfer function of a conventional imaging system changes with misfocus, but not in a manner that is easy for DSP to extract the desired information. For example, nulls appear in the OTF of a conventional imaging system as it moves out of focus, but they are not periodic, and the misfocus information is not easily extracted. In the example shown in Fig. 5, the OTF is designed to have a peak in spatial frequencies that moves with misfocus. So, to determine the misfocus of, or the distance to, an object in the scene, the peak is found for the spatial frequency spectrum of that portion of the image containing the object of interest. ${ }^{11,12}$ Figure 5 shows the lag, or ambiguity function for this system, and the OTF of the system for objects at two different distances. When an object is imaged by the modified optical system (if the object has a reasonable band of spatial frequencies) the peak that appears in the spatial frequency spectrum of the image will have a peak at the spatial frequency that is determined by the range of the object. Figure 6 shows the object scene used in an experiment. Model vehicles were placed on a painted roadbed. A cluster of pixels around the central pixel for the object must be used in the signal processing to determine the spatial frequency peak. The resulting information can be displayed as a range map, and Fig. 7 shows a densitycoded range map of the scene in Fig. 6. 


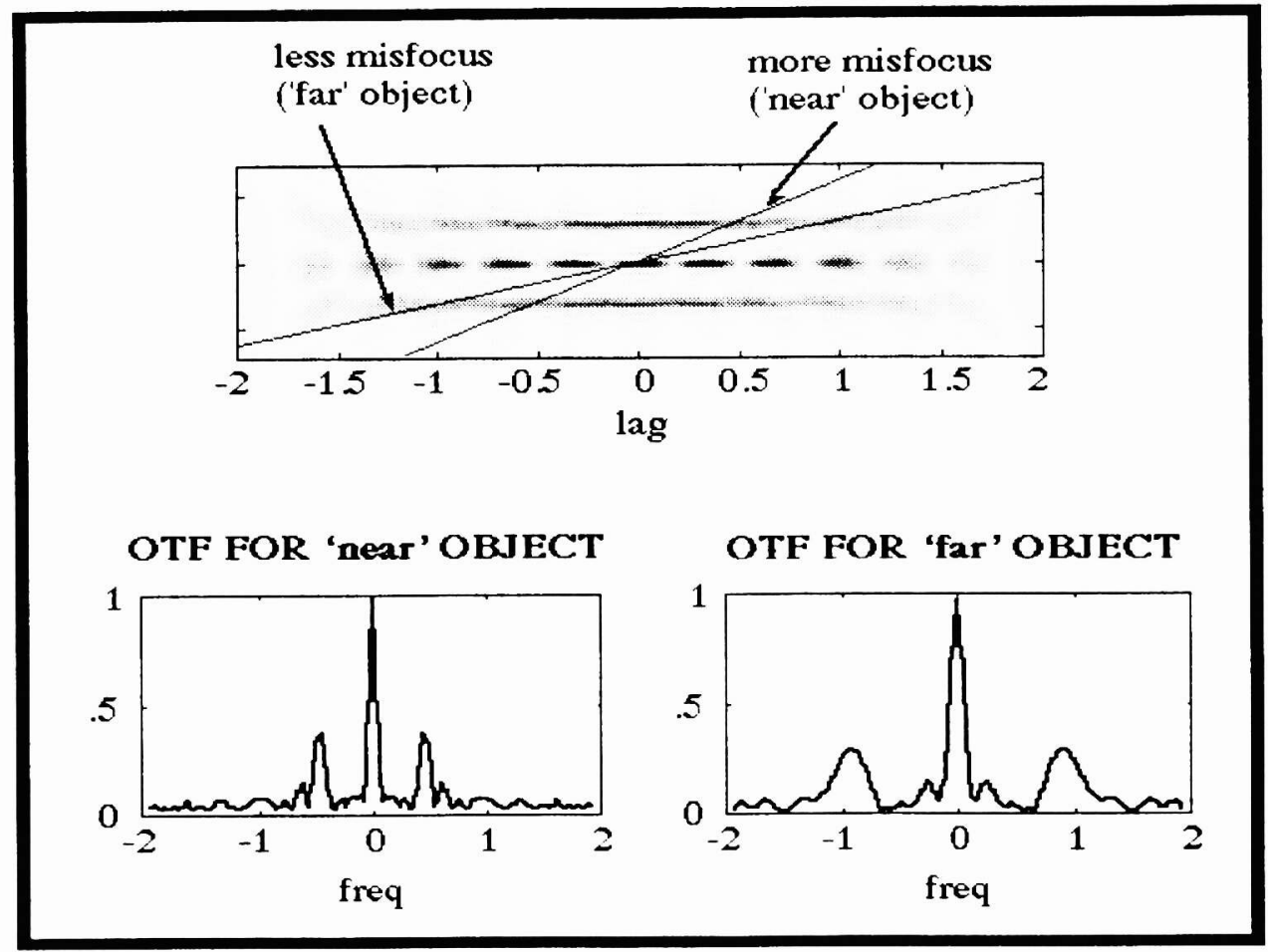

Fig. 5. The effect of the misfocus, or range of the object on the OTF for a hybrid digital-optical system that was designed to "image" range.

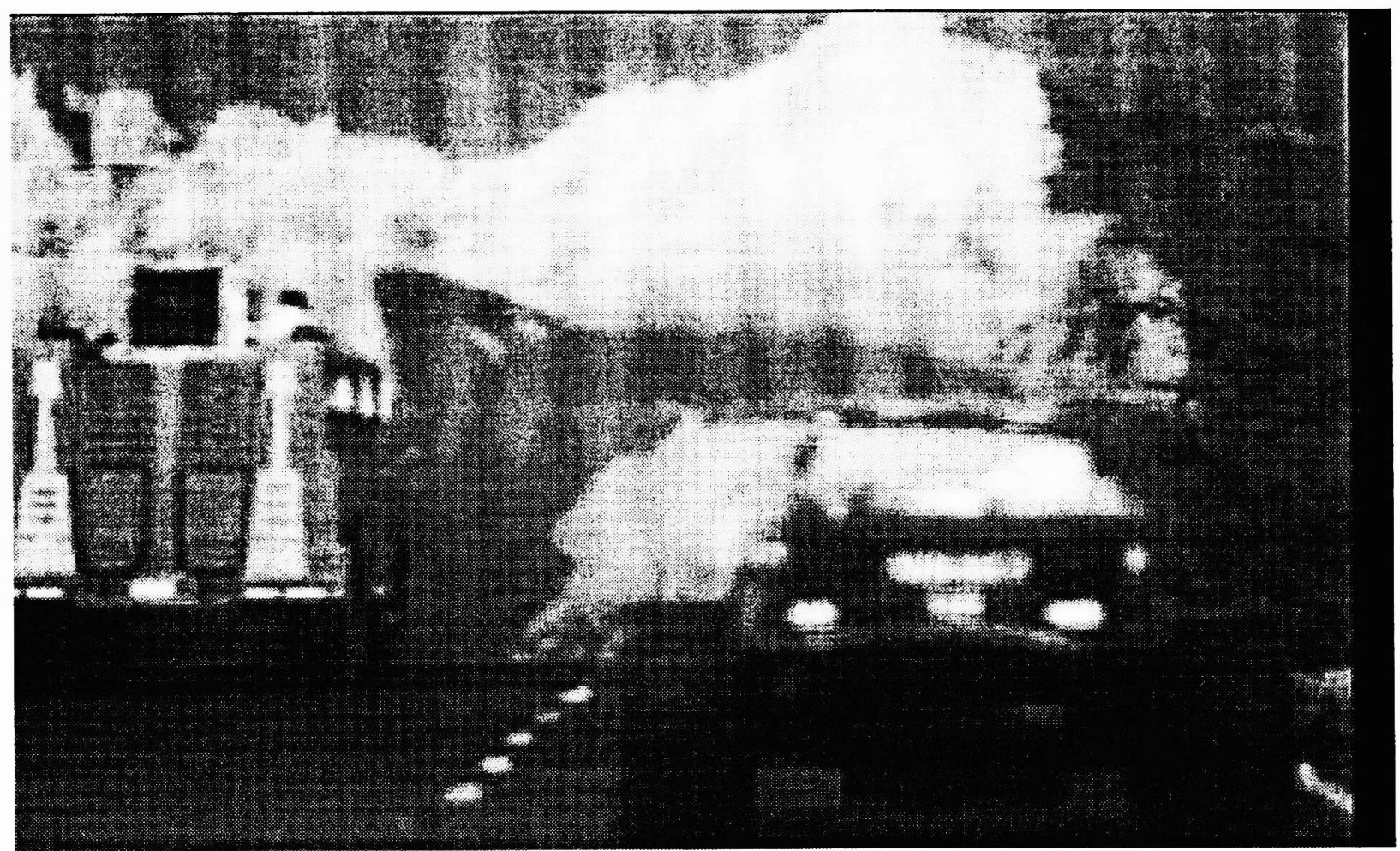

Fig. 6. Two models on a painted roadbed 

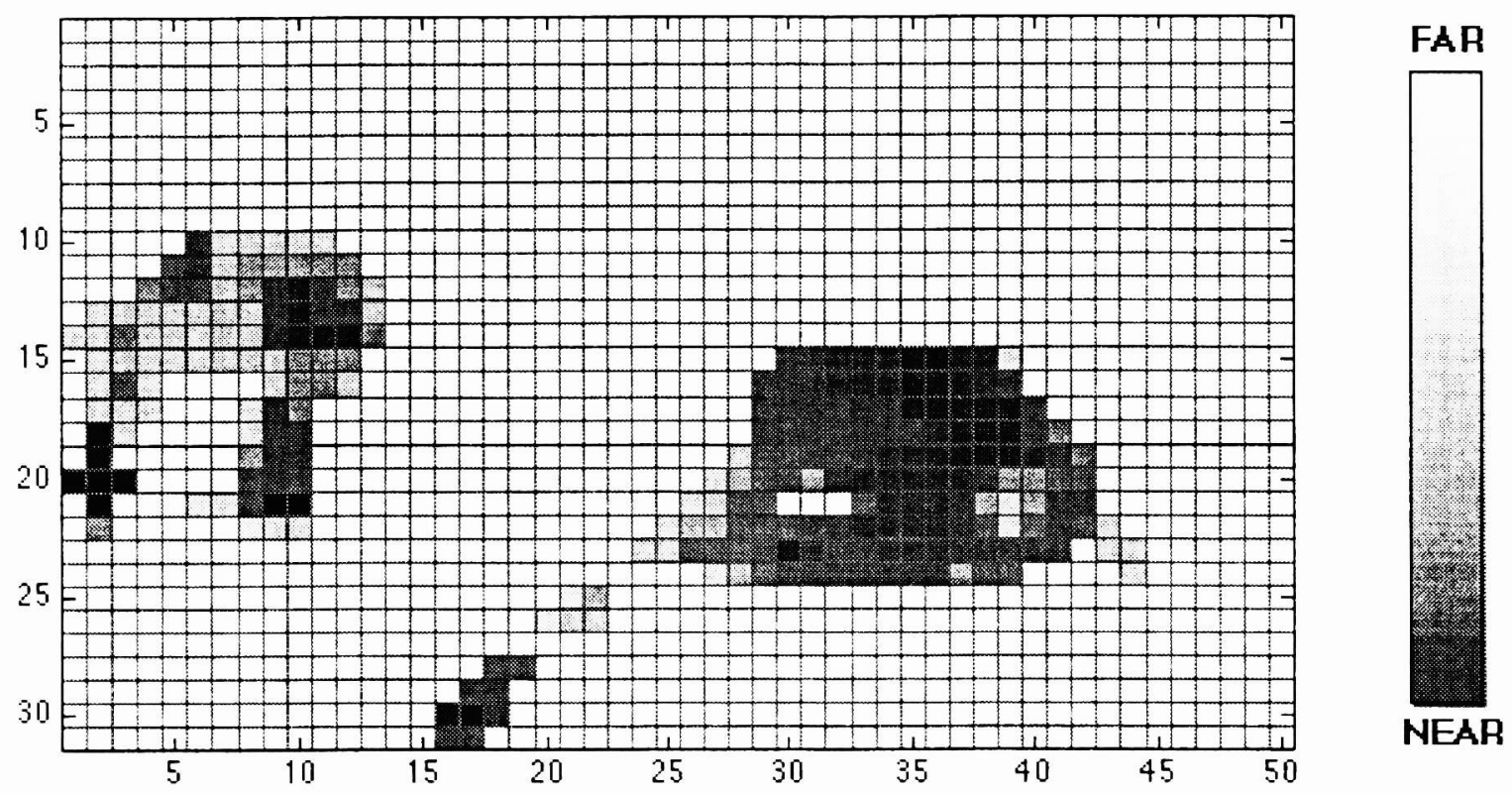

Fig. 7. Range map for the scene in Fig. 6.

\section{NEW PARADIGM FOR DESIGN OF IMAGING SYSTEMS}

The hybrid optical/digital system described in Section III has a very large depth of field. Alternatively, it can he considered to have a very large depth of focus. This means that it not only is tolerant of misfocus, but also of focus-related aberrations. For example, chromatic aberration, being a difference in focus position for different colors, can be tolerated because the focal point of each color can be extended until they all overlap. ${ }^{13}$ An example of this is shown in Fig. 8 , where the image in Fig. 8(a) was formed with a conventional imaging system, and the image of Fig. 8(b) was formed by a hybrid system that was designed to increase the depth of focus. The object was an Air Force test pattern, and blue was in best focus. Figure 9 shows images of the center part of the test pattern. Figure 10 shows the numeral "3" in the test pattern. Another experiment showed the use of wavefront coding for color correction in a microscope. ${ }^{14}$ 


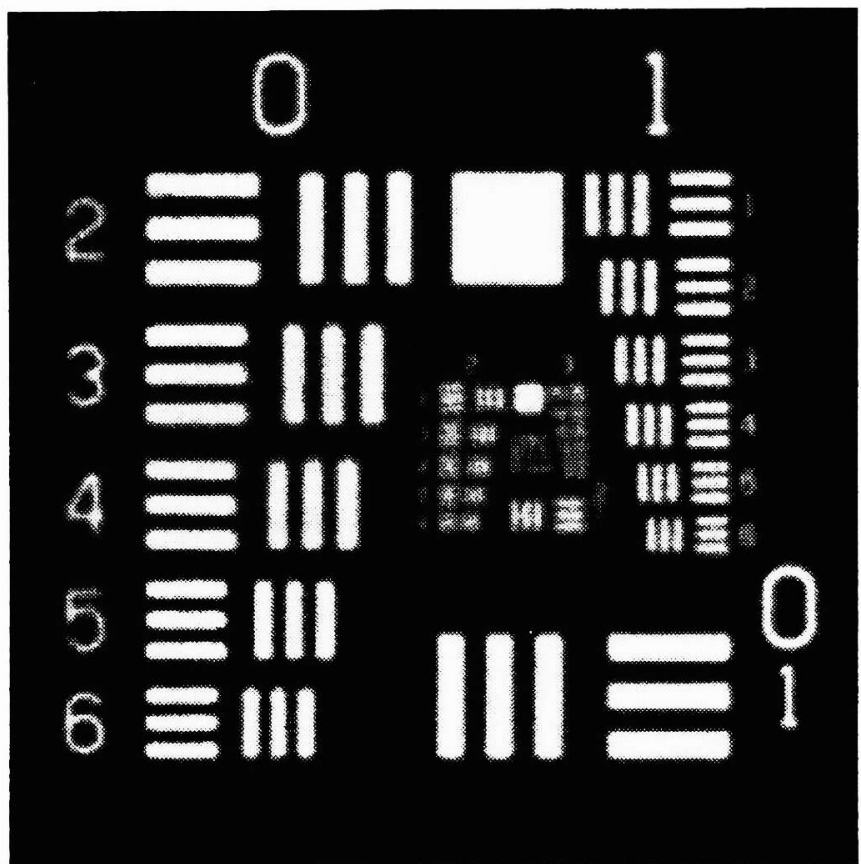

(a)

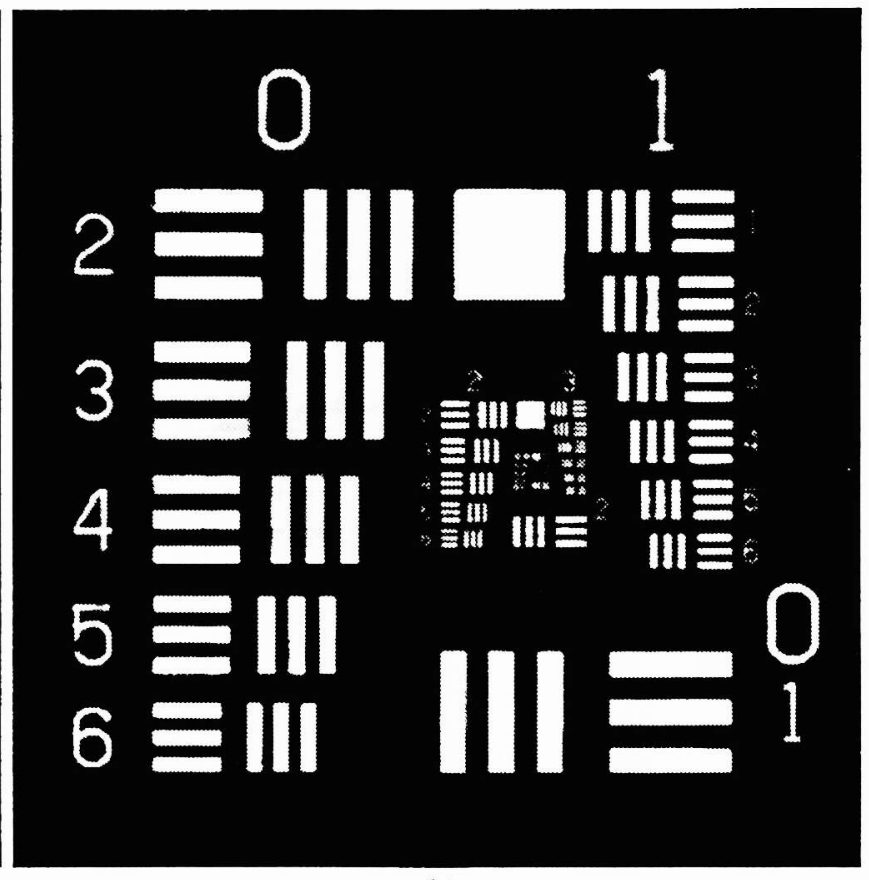

(b)

Fig. 8. A color image of an Air Force resolution test chart showing the images taken with (a) conventional imaging system, and (b) hybrid digital/optical imaging system.

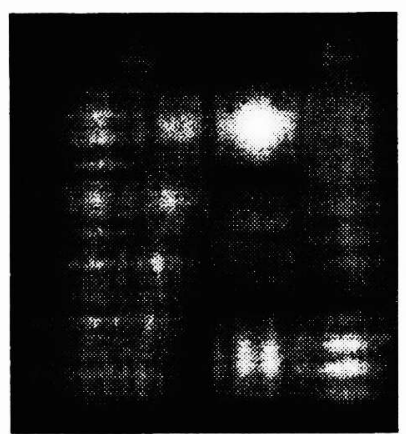

Red

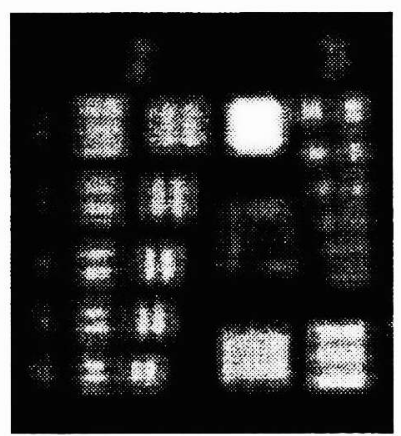

Green

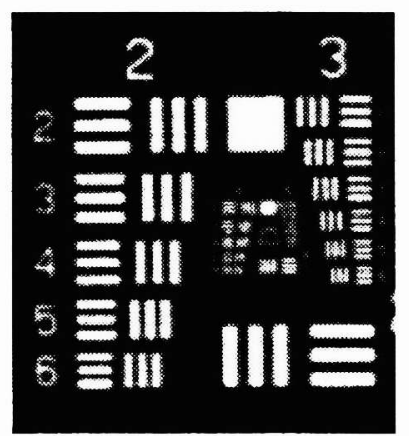

Blue

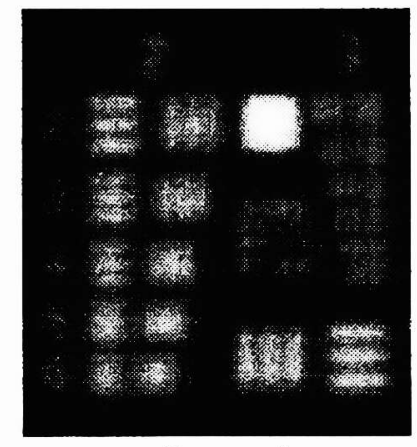

Composite

(a) Conventional System

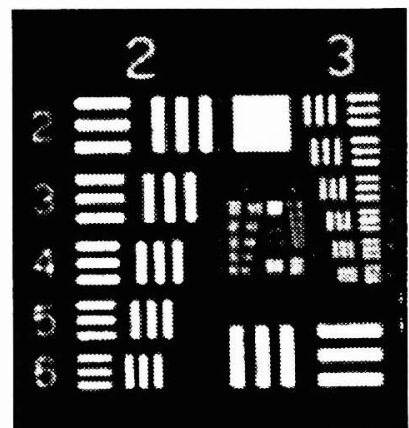

Red

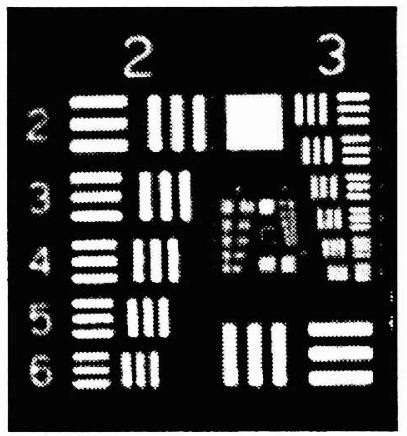

Green

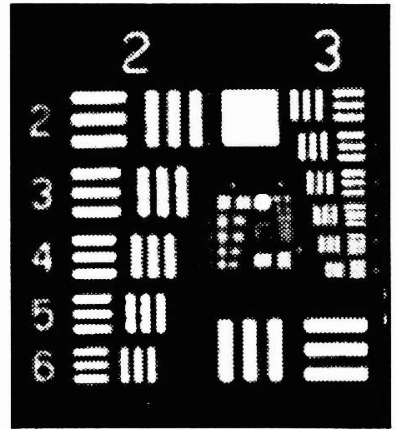

Blue

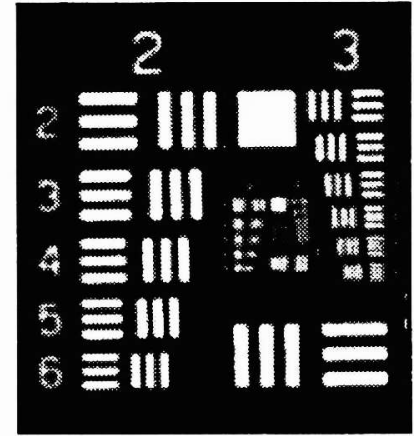

Composite

(b) Hybrid Digital/Optical Imaging System

Fig. 9. Center portion of the images of Fig. 8. 

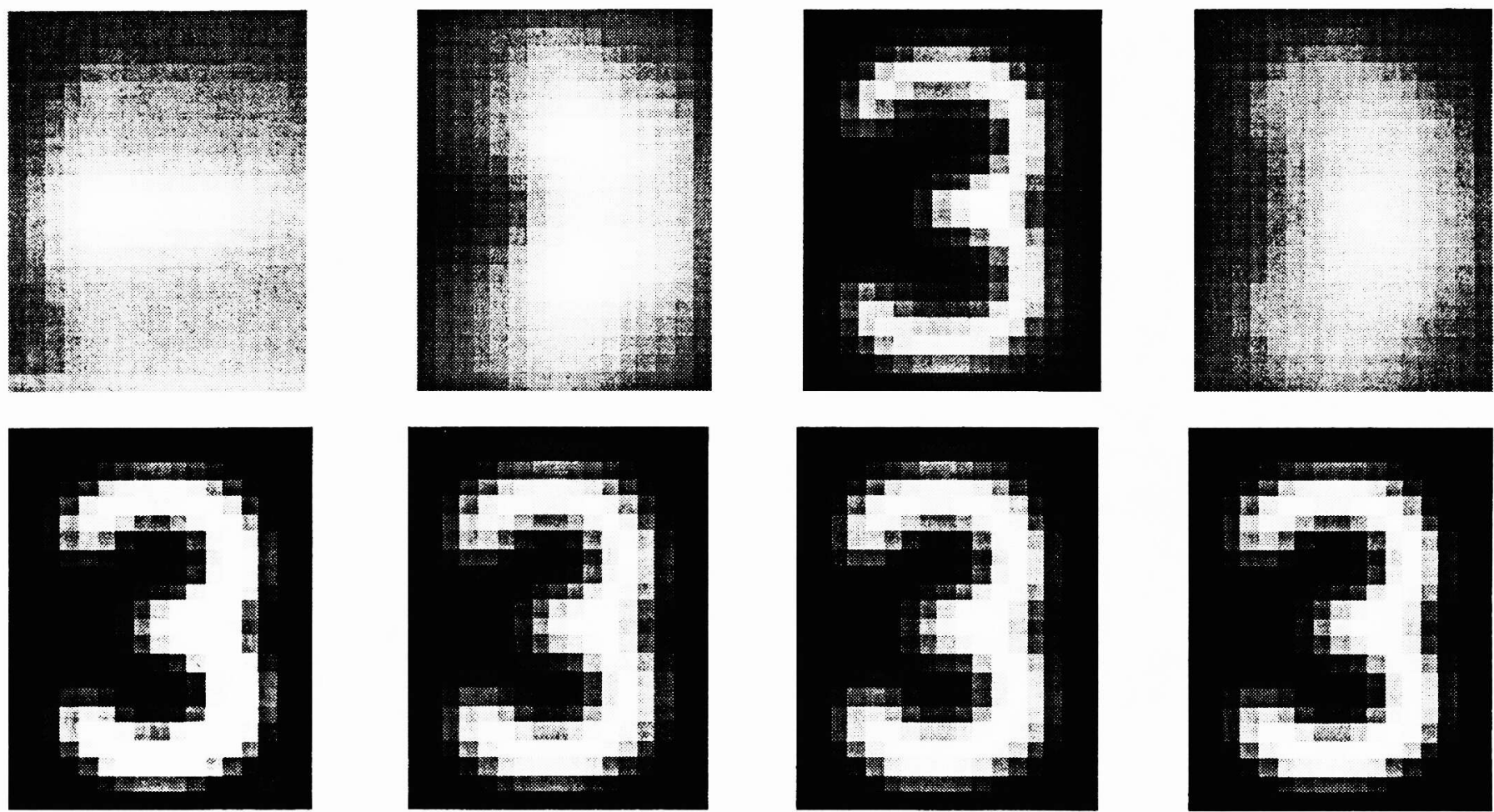

Green

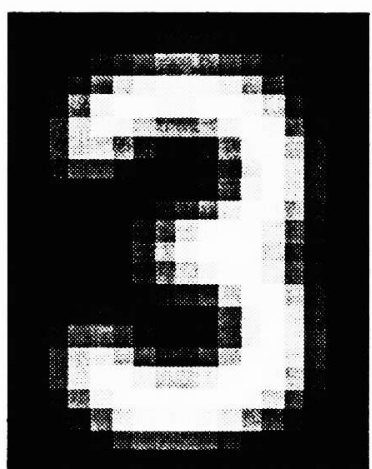

Blue

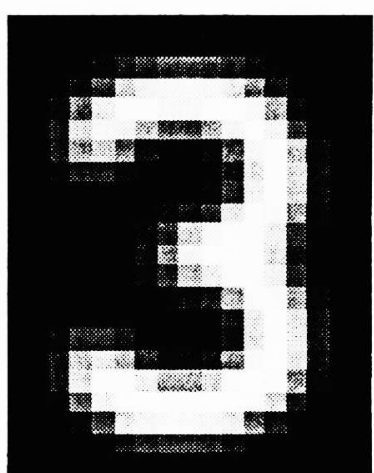

Composite

Fig. 10. Images of the numeral 3, showing the effects of axial chromatic aberration with a conventional imaging system (top). and a hybrid digital/optical system (bottom).

Curvature of field can be tolerated because, if the depth of focus is extended, a flat detector array can be fit into the curved, but much thicker focal region. This means that when balancing aberrations in the design of an imaging system, the focus-related aberrations can be accommodated by the combination of a waveplate that extends the depth of focus of the optics, and signal processing to restore the optical transfer function. The other aberrations are accommodated for as in normal lens design. but without having to be particularly concerned with the reduction of focus-related aberrations in the glass. As an extreme example of how the optical system can be simplified by use of wavefront coding and DSP, an acrylic single-element hybrid optical/digital imaging system has been designed that has a F/3, $3 \mathrm{~mm}$ lens with a $30^{\circ}$ field. ${ }^{15}$ This lens is shown in Fig. 11. Without the wavefront coding, the lens would have $12 \lambda$ of Petzval curvature.

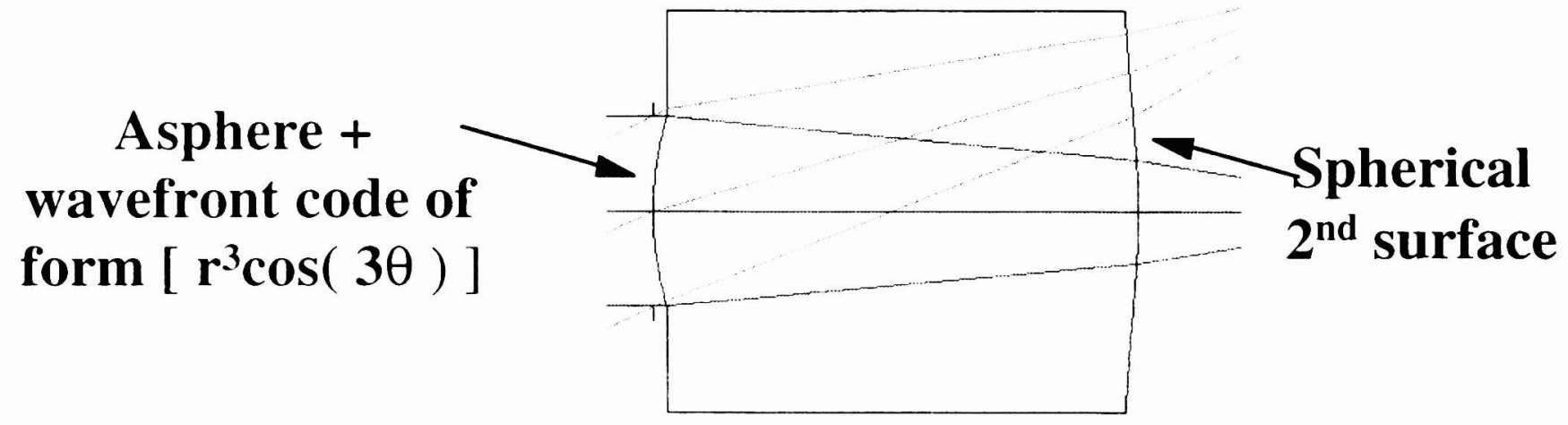

Fig. 11. A single-element hybrid imaging system with wavefront coding to extend the depth of focus and signal processing w restore the OTF. 


\section{CONCLUSIONS}

Those people who understood optics, linear systems, and Fourier theory made major contributions to optics that led to "Fourier optics" and "modern optics." Similarly, those who understand optics and the work that has been done in digital signal processing in the last few decades will have a tremendous impact on digital imaging systems. The required signal processing will be readily available in cameras that have electronic image detection arrays. The decreasing cost of the signal processing will make it practical to replace several conventional optical elements with fewer wavefront-coded optical elements and inexpensive signal processing. Already, it has been shown that hybrid optical/digital imaging systems can be built that have unique imaging characteristics. In these systems, the image acquisition is done by optics and a detector array, and the signal processing is done by digital signal processing. Future digital imaging systems will have capabilities far beyond what today's imaging systems can do and at lower cost.

\section{REFERENCES}

1. E. L. O’Neill, Introduction to Statistical Optics, Addison-Wesley, Reading, Mass., 1963.

2. W. T. Cathey, B. R. Frieden, W. T. Rhodes, and C. K. Rushforth, "Image gathering and processing for enhanced resolution," J. Optical Soc. of Am. 1A, pp. 241 - 250, 1984.

3. A. Papoulis, "Ambiguity function in Fourier optics," J. Optical Soc. of Am. 64, pp. 779 - $788,1974$.

4. K. Brenner, A. Lohmann, and J. Ojeda-Castaneda, "The ambiguity function as a polar display of the OTF," Optics Communications 44, pp. 323- 326, 1983.

5. E. R. Dowski and W. T. Cathey, "Extended depth of field through wavefront coding," Applied Optics 34, pp. 1859 1866, 1995.

6. W. W. Rihaczek, Principles of High Resolution Radar, McGraw-Hill, New York, 1969.

7. J. van der Gracht, E. R. Dowski, M. G. Taylor, and D. M. Deaver, "Broadband behavior of an optical-digital focusinvariant system," Optics Letters 21, pp. 919 - 921, 1996.

8. S. Bradburn, E. R. Dowski, and W. T. Cathey, "Realizations of focus invariance in optical-digital systems with wavefront coding, Applied Optics 36, 9157 - 9166, 1997.

9. E. R. Dowski, "An Information Theory Approach to Three Incoherent Information Processing Systems", Signal Recovery and Synthesis V, OSA Technical Digest Series, pp. 106-108, March, 1995.

10. E. R. Dowski and W. T. Cathey, Single-lens single-image incoherent passive-ranging systems," Applied Optics 33, pp. $6762-6773,1994$.

11. G. E. Johnson, E. R. Dowski, and W. T. Cathey, "Passive Ranging for Acquisition of Range Images: Application to Longitudinal Vehicle Control and Warning Systems," International Transportation Conference, Chicago, September 1997

12. G. E. Johnson, E. R. Dowski, and W. T. Cathey, "Passive ranging through wavefront coding: information and application," Applied Optics, submitted.

13. H. B. Wach, E. R. Dowski, and W. T. Cathey, "Control of chromatic focal shift through wavefront coding," Applied Optics 34, pp. 1859 - 1866, 1995.

14. S. C. Tucker, W. T. Cathey, and E. R. Dowski, Extended depth of field and aberration control for inexpensive digital microscope systems," Optics Express 4, pp. 467 - 474, 1999.

15. E. R. Dowski, "Simplified lens design for digital imaging systems," Optical Society of America 1998 Annual Meeting, Baltimore, Maryland, 5 Oct. 1998. 